\title{
Dependence of Solid Oxide Fuel Cell Electrode Microstructure Parameters on Focused Ion Beam - Scanning Electron Microscopy Resolution
}

\author{
Takaaki Shimura*a $^{* a}$ Zhenjun $\operatorname{Jiao}^{a}$, Naoki Shikazono ${ }^{a, b}$ \\ a. Institute of Industrial Science, The University of Tokyo 4-6-1 Komaba, Meguro-ku, Tokyo 153-8505, Japan \\ b.CREST, JST, 4-6-1 Komaba, Meguro-ku, Tokyo 153-8505, Japan
}

\begin{abstract}
Dependence of microstructure parameters on the resolution of focused ion beam-scanning electron microscopy (FIB-SEM) images was evaluated for nickel-yttria stabilized zirconia (Ni-YSZ) electrode of solid oxide fuel cell (SOFC). Three dimensional microstructure was reconstructed by FIB-SEM images at voxel size of $3 \mathrm{~nm}$ which is the highest resolution among the reported SOFC researches. Volume fraction, triple phase boundary (TPB) density and specific surface areas were calculated with various resolutions. Dependences of TPB density and specific surface areas on resolution were clearly shown. Changes of these parameters at high resolution were attributed to the surface roughness caused by the image drift in the scanning electron microscopy imaging. Accurate vales of TPB density and specific surface area were estimated by extrapolation from several values at coarser resolutions and compared with those at typical resolutions which are normally applied in conventional microstructure analysis of SOFC electrodes. The result indicates that the dependence on resolution should be taken into account for the quantitative analysis of microstructure parameters.
\end{abstract}

Keywords: solid oxide fuel cell, 3D reconstruction, resolution, focused ion beam - scanning electron microscopy, image processing;

\section{Introduction}

Electrode performance of solid oxide fuel cells (SOFC) are mainly dominated by the ionic diffusion and the electrochemical reaction rates at the triple phase boundaries (TPB) or at the surface. It is widely known that the electrode performances are significantly affected by the electrode microstructure. Therefore, three-dimensional (3D) reconstruction techniques have been widely applied in the evaluation of electrode

Email address: tshimura@iis.u-tokyo.ac.jp (Takaaki Shimura*a) 
microstructures. Several studies on 3D morphology observation by nano X-ray tomography for Nickel-Yttria stabilized zirconia (Ni-YSZ) electrodes have been reported [1-5]. This technique has the advantage of nondestructive observation. However, its resolution is limited to around $50 \mathrm{~nm}[1]$. Another technique for 3D observation is the dual beam focused ion-scanning electron microscopy (FIB-SEM) [6-13]. Typical image pixel sizes for the FIB-SEM reconstruction are around several tens micrometers, and the dependence of microstructure parameters on resolution has been investigated by several researchers. Hutzenlaub, et al., [14] changed the resolution of reconstructed microstructure from FIB-SEM and calculated the corresponding microstructure parameters of polymer electrolyte membrane fuel cell cathode. It was found that there were strong dependences of specific surface area and conductivity on resolution, whereas volume fractions showed only a weak dependence. Klingele, et al., also considered the influence of FIB slice pitch on the gas diffusivity of the gas phase and the electronic conductivities of the solid phase [15]. However, investigated parameters have been limited to volume fractions and conductivities in most cases. In Ni-YSZ anode of SOFC, electrochemical reactions take place at the TPB. Thus, TPB density is considered to be one of the most important parameters for the electrode performance. However, the reliability of estimated TPB density values with different resolutions has been rarely investigated. In order to clarify the dependence of microstructure parameters of Ni-YSZ anode on resolution, sensitivity analysis should be conducted with higher resolution of nm scale.

Furthermore, in order to analyze the resolution sensitivity, reliable imaging methods which ensure the quality of segmented images need to be established. The most common method for image segmentation is the thresholding. Joos, et al., evaluated the microstructure of $\mathrm{La}_{0.58} \mathrm{Sr}_{0.4} \mathrm{Co}_{0.2} \mathrm{Fe}_{0.8} \mathrm{O}_{3-\delta}$ electrode based on FIB-SEM technique [16] by thresholding the gray scale values. They found that volume fraction has larger sensitivity than the surface area to the threshold values of the images. Strong dependence of volume fraction on threshold value was also reported by Chen, et al. [17]. Furthermore, during image processing, the interface shape can be easily deformed when an arbitrary grayscale threshold value is simply applied for segmentation, which is due to the transient brightness distribution at the interface. To prevent the deformation of phase interface shape, intensification of edge lines is considered to be one of the solutions. Such approach has been proposed by Harris, et al., [18] in which the image processing was conducted by watershed algorithms.

In this study, in order to evaluate the accuracy of microstructure parameters, dependence of Ni-YSZ microstructure on resolution was investigated using high resolution FIB-SEM. Three dimensional microstructures of Ni-YSZ anode were reconstructed with a pixel size of $3 \mathrm{~nm}$, which is by far the highest resolution 
among the reported studies on 3D reconstructions of SOFC electrodes. In addition, an image processing method with the use of edge lines was introduced to improve the image quality.

\section{Methods}

\subsection{Sample preparation}

Electrolyte supported cell with Ni-YSZ composite anode after $100 \mathrm{~h}$ operation with a current density of $0.2 \mathrm{Acm}^{-2}$ at $800^{\circ} \mathrm{C}$ was used as a sample. Details of the experimental procedure can be found in Ref. [19]. After the experiment, a piece of the cell was filled with epoxy resin (Struers Epofix), and polished with sand paper and cross section polisher (JEOL SM-09020CP).

\subsection{FIB-SEM observation}

FIB-SEM (SMF-1000, SII Nanotechnology) was used in this study to obtain high resolution images. This machine has an orthogonal alignment of SEM and FIB columns. This enables shorter SEM working distance of $2 \mathrm{~mm}$ and smaller FIB slicing distance of $2 \mathrm{~nm}$ as a minimum pitch. In this study, SEM resolution and FIB slicing pitch were set at $3 \mathrm{~nm}$. To our best knowledge, this is the highest resolution among the studies based on FIB-SEM reconstruction of SOFC electrodes. One of the cross-sectional images is shown in Fig. 1 (a). Images were taken at $1 \mathrm{kV}$ acceleration voltage with upper secondary-electron detector.

\subsection{Image processing}

FIB-SEM images were ternarized into Ni, YSZ and pore phases through several procedures. First, raw images were aligned by stackreg algorithm of ImageJ [21]. The target region for reconstruction was then trimmed. Amplified image after trimming is shown in Fig. 1 (b). The edges of each phase were detected by ImageJ as shown in Fig. 1 (c). At this step, edge line consists of about ten white pixels in width, partially including the noises of several black pixels. In order to erase those noises within the edge lines, edge lines were skeletonized using ImageJ as shown in Fig. 1 (d). Phase segmentation processes were accomplished by in-house algorithms. Skeletonized images were then merged with original trimmed images as shown in Fig. 1 (e). In this process, grayscale values of the trimmed image were reduced to ensure that the skeletonized edge has the highest grayscale value. As a consequence, merged images consists of several closed regions surrounded by the skeletonized edge lines. Then, mean grayscale value was calculated for each closed region. Based on the calculated mean grayscale values, phase identification was conducted by comparing the given threshold values. After phase identification, each closed region was filled with a specific 
grayscale value as shown in Fig. 1 (f). After image segmentation, unnatural segmentations resulted from disconnected skeletonized edge lines and remained voids within the resin were corrected manually. After all corrections, skeletonized edge lines were erased by replacing them with the grayscale value of the adjacent pixels. Finally, erroneous geometries smaller than 1 pixel were filled with the surrounding brightness value and image processing was completed as shown in Fig. $1(\mathrm{~g})$. 


$$
\mathbf{L}
$$




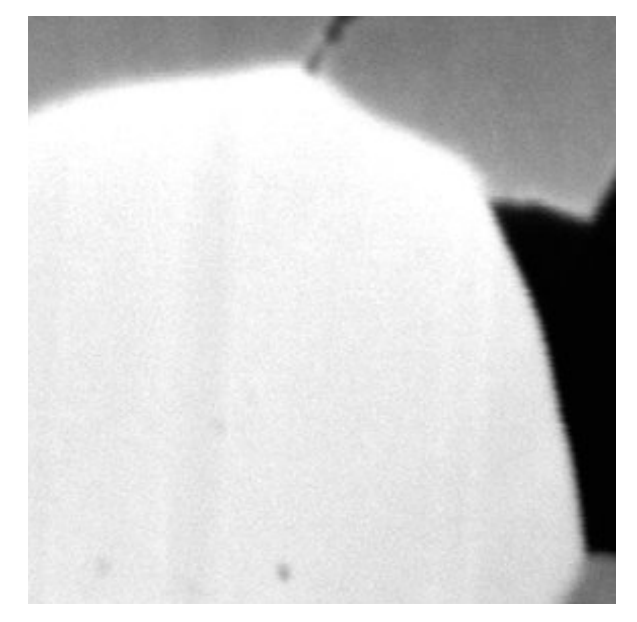

(b)

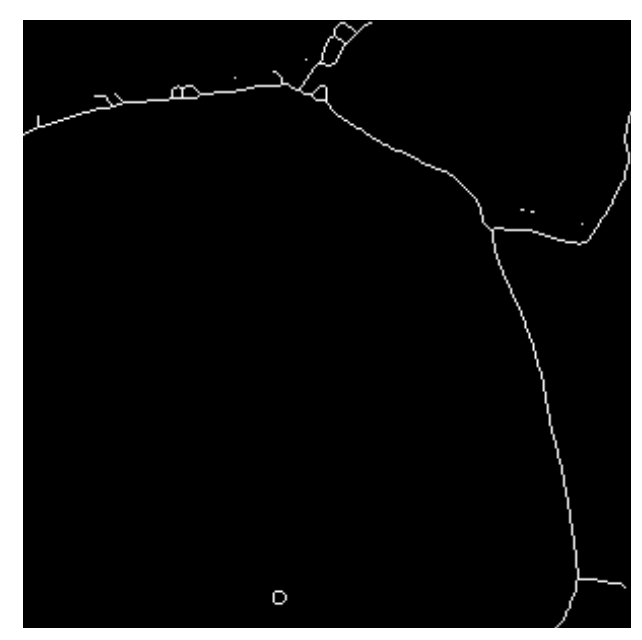

(d)

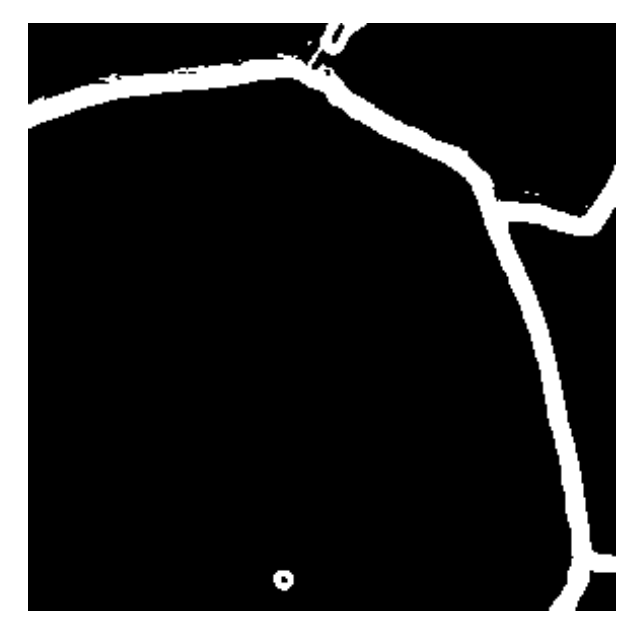

(c)

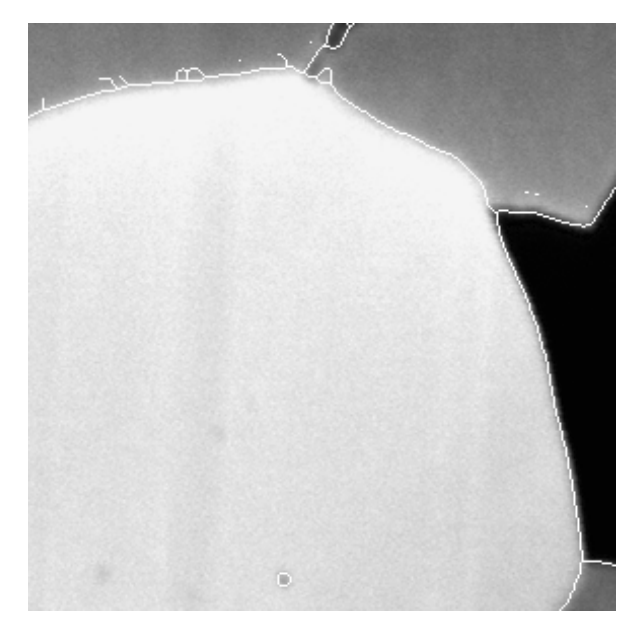

(e) 


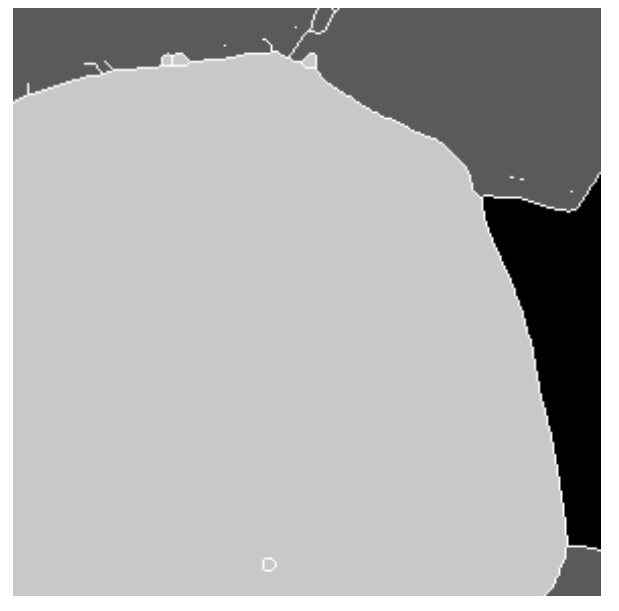

(f)

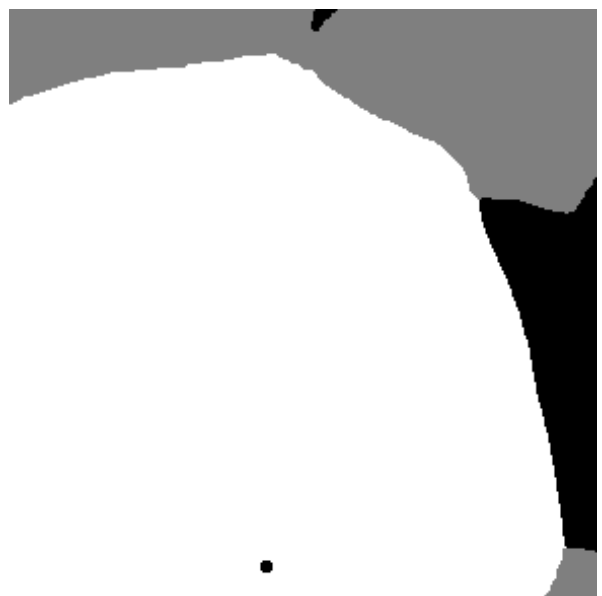

(g)

Figure 1: Sequence of the image processing. (a) Original image after alignment and trimming. Bright and gray phases correspond to Ni and YSZ, respectively. (b) Magnification of a part of (a). (c) Edge detected from (b). (d) Skeletonized edge. (e) Merged image of (b) and (d). (f) Segmented image, and (g) final image. 
After image processing, ternarized images were resized with various resolutions as shown in Fig. 2.

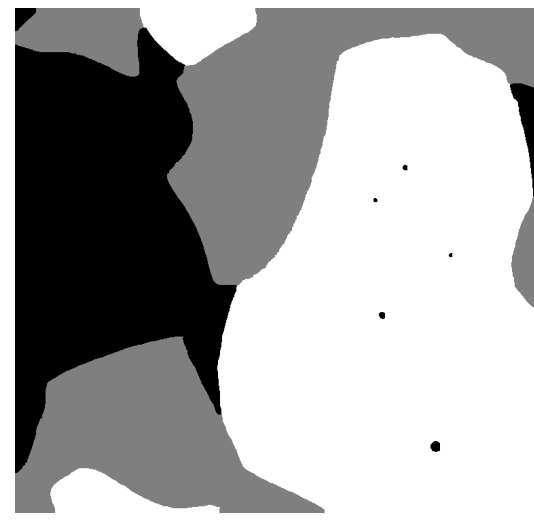

(a)

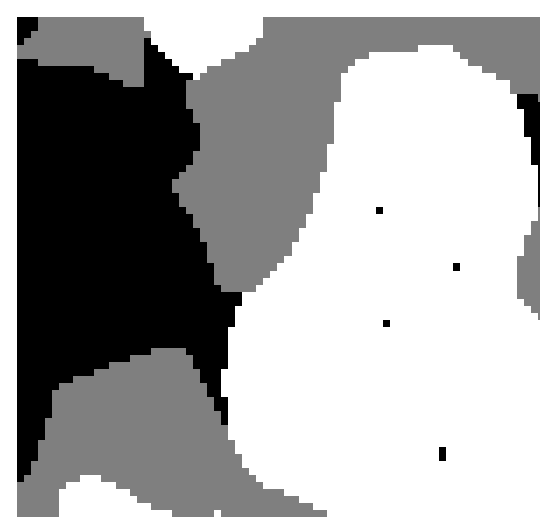

(d)

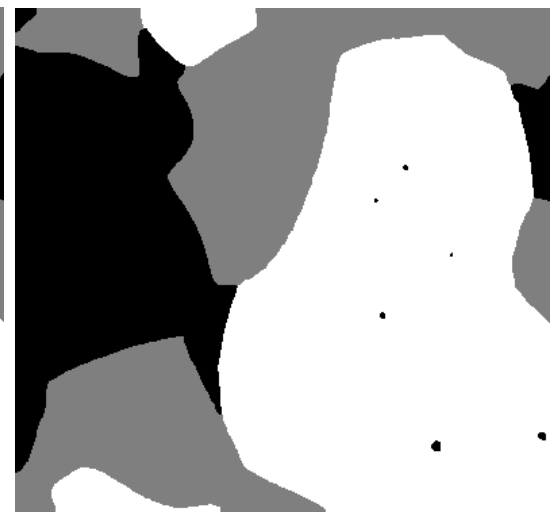

(b)

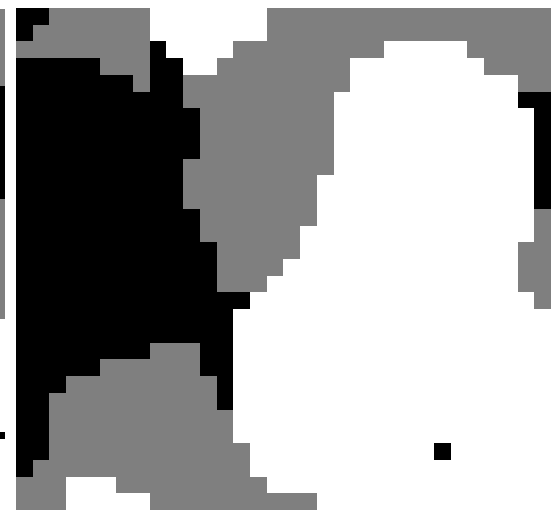

(e)

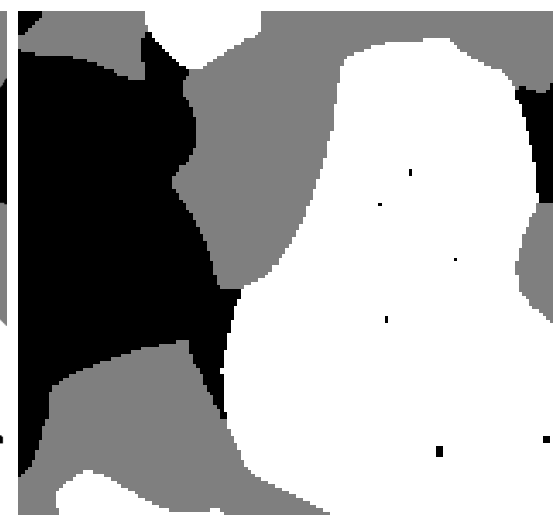

(c)

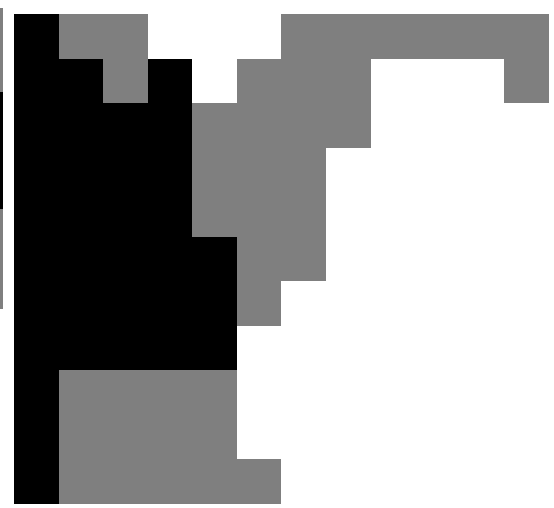

(f)

Figure 2: Resized images. Number of pixels and resolution are shown below each of the image. (a) $788 \times 744 \times 896(3.000$ $\mathrm{nm} /$ pixel $)$, (b) $396 \times 373 \times 450(5.970 \mathrm{~nm} / \mathrm{pixel})$, (c) $156 \times 147 \times 177(15.15 \mathrm{~nm} / \mathrm{pixel})$, (d) $76 \times 71 \times 86(31.11 \mathrm{~nm} / \mathrm{pixel})$, (e) $32 \times 30 \times 36(73.88 \mathrm{~nm} /$ pixel $),(f) 12 \times 11 \times 13(197.0 \mathrm{~nm} /$ pixel $)$

\subsection{Parameter calculation}

Based on the ternarized images, 3D microstructures were reconstructed. Volume fraction of each phase was calculated by counting the number of voxels. TPB density was calculated by three different methods, i.e. edge length, mid-point and centroid methods [22]. Specific surface areas were calculated by the marching cube method as described in Ref. [23].

\section{Results}

Microstructure parameters calculated from ternarized images are shown in Fig. 3. It can be seen from the figure that the volume fractions of three phases were almost constant regardless of the resolution. The 
trends of TPB densities were similar for the three calculation methods. Significant change of TPB densities were found for the pixel sizes below $20 \mathrm{~nm}$ as shown in Fig. 3 (b). Between pixel sizes from $20 \mathrm{~nm}$ to 100 nm, TPB densities showed nearly linear dependence on resolution. From Fig. 3 (c), we can see that specific surface areas also shows large change below pixel size of $20 \mathrm{~nm}$. Beyond $20 \mathrm{~nm}$, they showed nearly linear trend as in the case for TPB. Thus, dependences of microstructure parameters on resolution, especially at high resolution, were revealed by the 3D reconstruction with higher resolution than the past literatures.

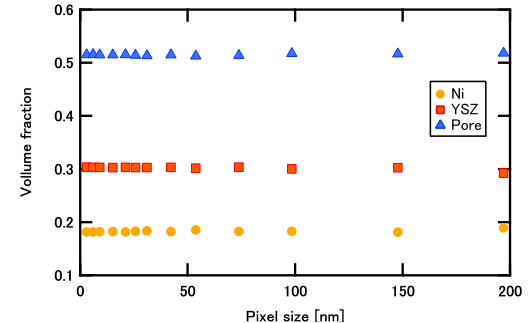

(a)

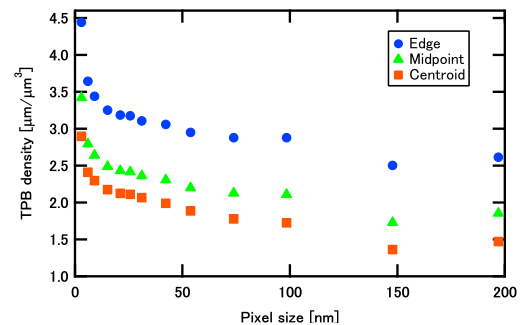

(b)

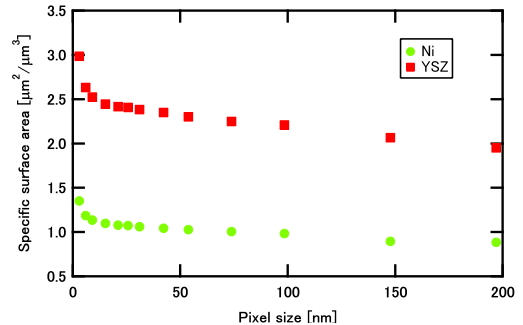

(c)

Figure 3: Calculated parameters from reconstructed structures against resolution. (a) Volume fraction, (b) TPB density and (c) specific surface area

\section{Discussion}

Weak dependence of volume fractions on resolution which is shown in Fig. 3 (a) is similar to what has been reported in Ref. [14]. On the other hand, large values of TPB density and specific surface areas were observed at high resolution as shown in Fig. 3. Magnified microstructures are shown in Fig. 4. As can be seen in Fig. 4 (b), TPB appears rough. The roughness at TPB corresponds to the surface roughness of $\mathrm{Ni}$ and YSZ. This roughness randomly repeated in the $\mathrm{z}$ direction of the structure, which corresponds to the stacking direction of the images. Such factitious roughness can be observed in the reconstructed structure of the original SEM images as shown in Fig. 4 (a), which indicates that there's a slight fluctuation in the original SEM images. This fluctuation can be attributed to the drift in every scanning of SEM, which may cause slight stretch or shrinkage of the SEM images. When the resolution was coarsened, roughness disappeared as shown in Fig. 4 (c)-(e). This implies that the increase of TPB density and specific surface areas at high resolution is caused by the roughness in the stacking direction of SEM images. The roughness of TPB in Fig. 4 (b) is estimated to be at most 5 pixels, i.e. $15 \mathrm{~nm}$. 


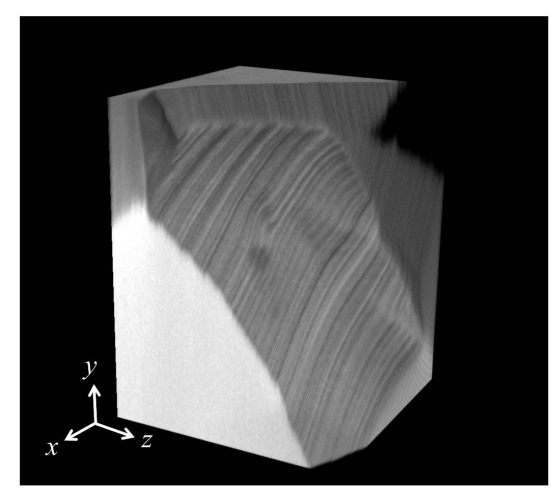

(a)

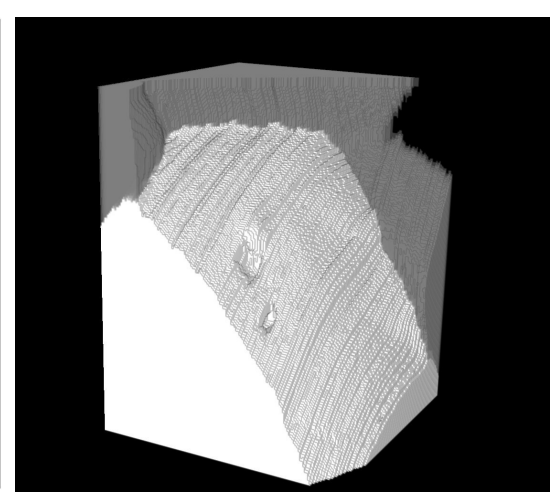

(b)

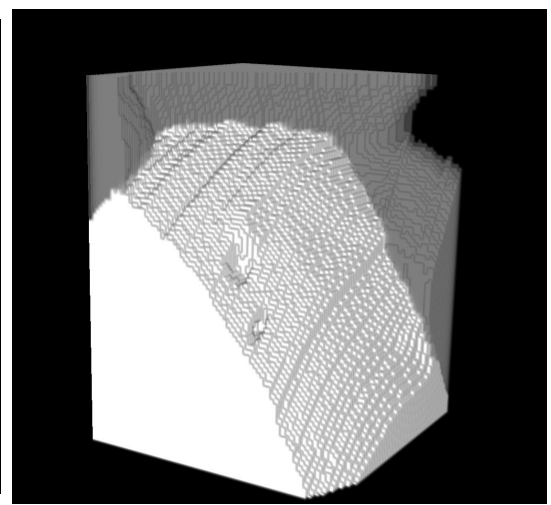

(c)

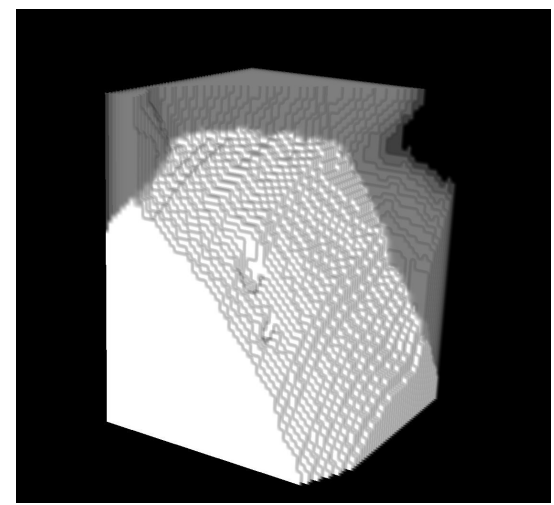

(d)

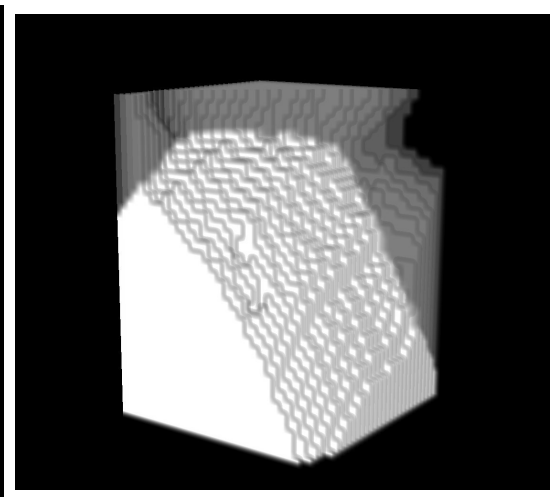

(e)

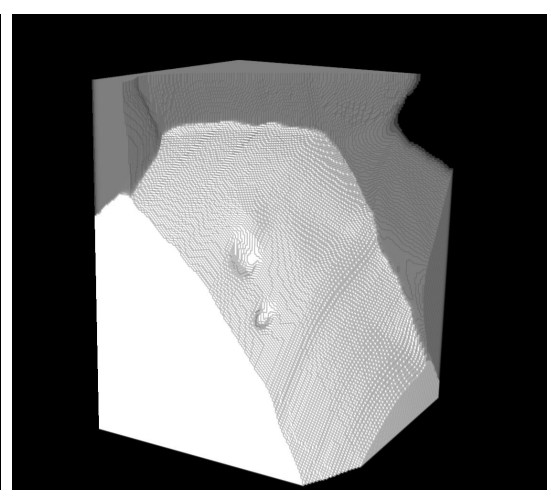

(f)

Figure 4: Visualization of a part of the structure $(0.45 \mu \mathrm{m} \times 0.60 \mu \mathrm{m} \times 0.45 \mu \mathrm{m})$. (a) Reconstruction from raw SEM images, (b)-(e) ternarized images at various voxel sizes ( $3 \mathrm{~nm}, 5.9697 \mathrm{~nm}, 9.0923 \mathrm{~nm}$ and $15.1538 \mathrm{~nm}$ ), and (f) reconstruction from ternarized images with $3 \mathrm{D}$ median filtering. White is $\mathrm{Ni}$, gray is YSZ, respectively. 
To evaluate the influence of factitious roughness on reconstructed structure, 3D median filtering was applied to series of ternarized images using ImageJ. Median filtering is a common tool to reduce the noise and to smoothen the images $[3,24,25]$. Radius of the $3 \mathrm{D}$ median filtering was set at 5 pixels according to the results of Gunda, et al., [25]. After 3D median filtering, images were ternarized again by repeating the image processing described in Section 2. Ternarized images with and without 3D median filtering are shown in Fig. 5. 


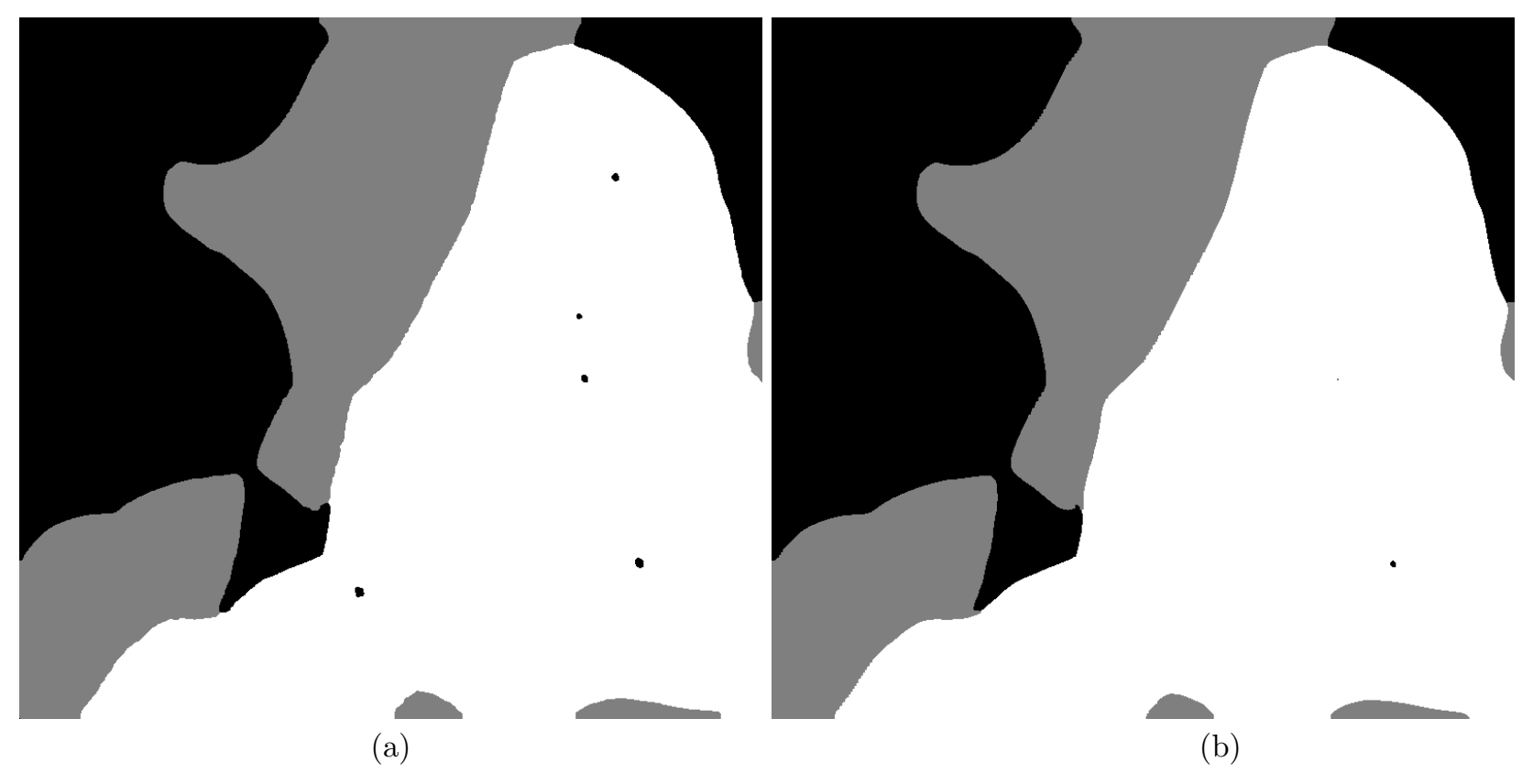




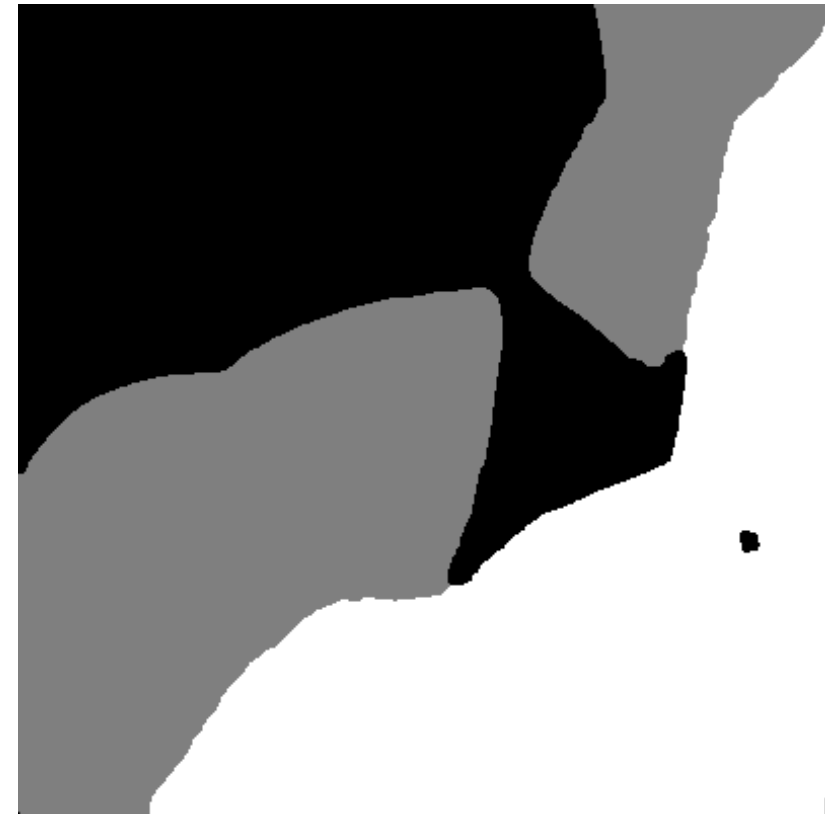

(c)

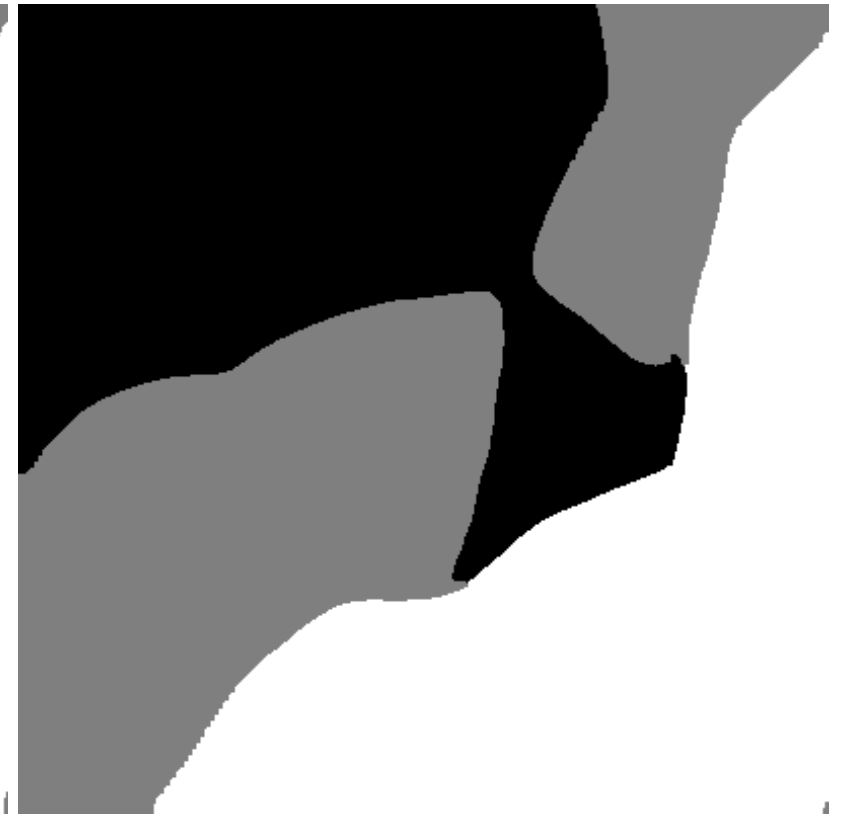

(d)

Figure 5: Comparison of images with and without 3D median filtering. (a) Ternarized image, (b) ternarized image with 3D median filtering, (c) enlarged image of the ternarized image, (d) enlarged image of the ternarized image with 3D median filtering 
As shown in Fig. 5 (a) and (b), whole shape became smoother after 3D median filtering. Reconstructed structure also became smoother after 3D median filtering as shown in Fig. 4 (f). Microstructure parameters calculated based on ternarized images with 3D median filtering are shown in Fig. 6. As shown in Fig. 6 (a), large TPB densities and specific surface area values found at high resolution were moderated compared with those in Fig. 3 (b). All parameters showed linear changes for the whole resolution range. These results also indicates that large TPB densities and specific surface area values found in Fig. 3 are originated from the factitious surface roughness of the reconstructed structures. The linear change of parameters observed from microstructure after 3D median filtering indicates that the dependence on resolution still remains in the smooth microstructure. At the same time, small pores disappeared (see Fig. 5 (a) and (b)) and local geometries around the TPB have changed (see Fig. 5 (c) and (d)). It must be noted that filtering has a possibility of losing local features of the original microstructure. The prediction of accurate values of microstructure parameters from values at several resolutions is proposed by Klingele et al. [15]. In this study, we also estimated the accurate values based on extrapolation of several sets of values at different resolutions.

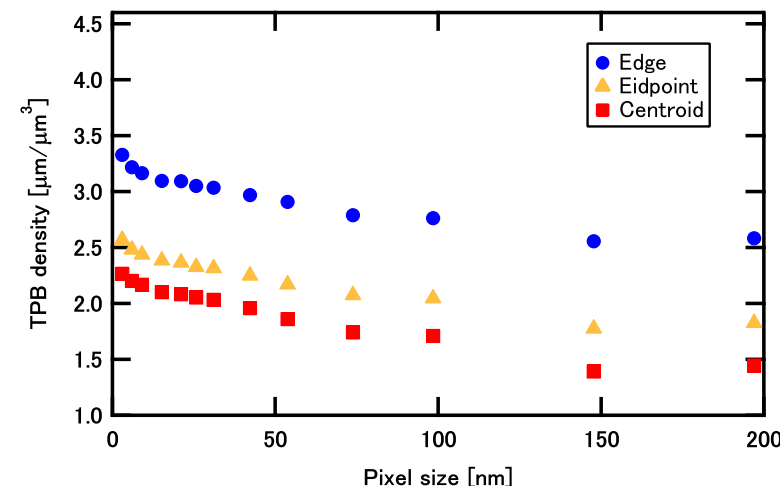

(a)

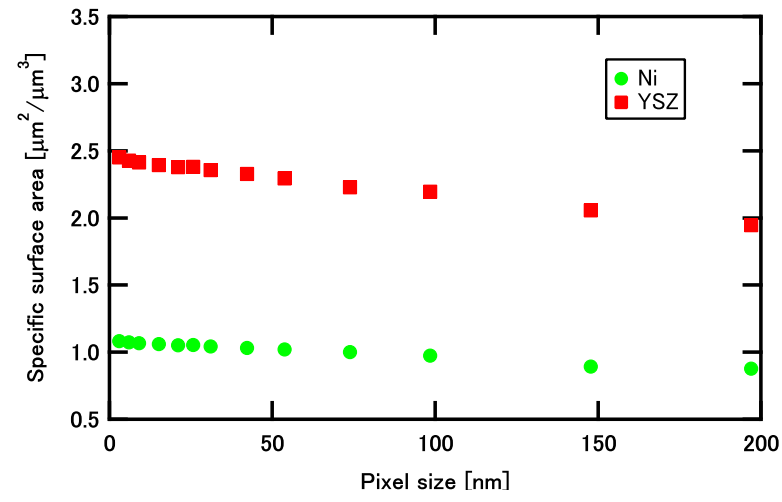

(b)

Figure 6: Calculated parameters from reconstructed structures after 3D median filtering against resolution. (a) TPB density, and (b) specific surface area

First, the reliability of extrapolation and the influence of roughness were evaluated using simple geometries. Perimeter lengths of two dimensional circular arc and an inclined square with tilt angle of $15^{\circ}$ were calculated at various resolutions. Circular arc and inclined square are chosen to investigate the effect of curvature variation. The diameter of the circle was $768 \Delta x$, where $\Delta x$ represents the original pixel size. The length of the square side was also set equal to $768 \Delta x$. In addition, roughness of $5 \Delta x$ pixels was randomly generated in the vertical direction. Roughness of $5 \Delta x$ pixels is determined from the ratio of the surface 
roughness to the diameter of Ni/YSZ interface in the real 3D microstructure. The circle with and without roughness are shown in Fig. 7 (a) and (b), respectively. Then, the images were resized to various pixel sizes. Perimeter lengths were calculated in the same way for the TPB calculation.
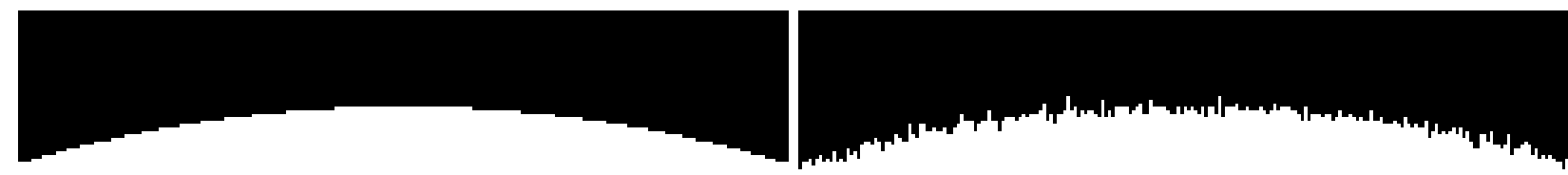

(a)

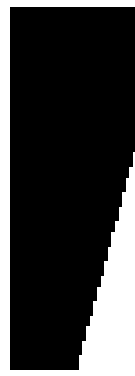

(c) (b)

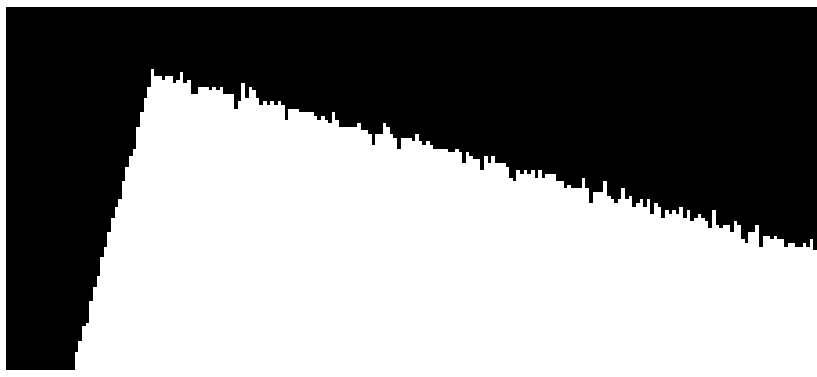

(d)

Figure 7: Part of reference images. (a) Smooth circle, (b) circle with roughness, (c) smooth square and (d) square with roughness.

In Fig. 8 (a) and (b), dependences of calculated perimeter lengths on resolution are shown. Calculated lengths are normalized by the analytical values and shown as relative values. As shown in Fig. 8 (a), for the circular arc with roughness, calculated length showed significant increase when the pixel size is smaller than $8 \Delta x$. It converged when the pixel size became larger than $8 \Delta x$. The asymptote of the circular arc length is almost identical to that of the circle without roughness. This implies that the influence of surface roughness can be eliminated by using coarser grid values. For the smooth arc without roughness, the error of the centroid method was about $1 \%$ when the pixel size is smaller than $16 \Delta x$. Thus, centroid method will give an accurate result provided that the resolution is high enough. At large pixel sizes, calculated arc length became constant. This is because the circle consists of single curvature. As shown in Fig. 8 (c), perimeter length of the square with roughness showed significant increase when the pixel size is below $8 \Delta x$. When the pixel size is larger than $8 \Delta x$, perimeter length gradually decreased due to the existence of sharp corner. This implies that the dependence on resolution is affected also by the curvature variation. The 
influence of roughness was largely reduced again when the pixel size is larger than $8 \Delta x$. For the square without roughness, when the pixel size is less than $16 \Delta x$, the error of the centroid method was less than $1 \%$. Thus, accurate values can be estimated by extrapolating the data from the coarser resolutions where the influence of roughness is negligible.

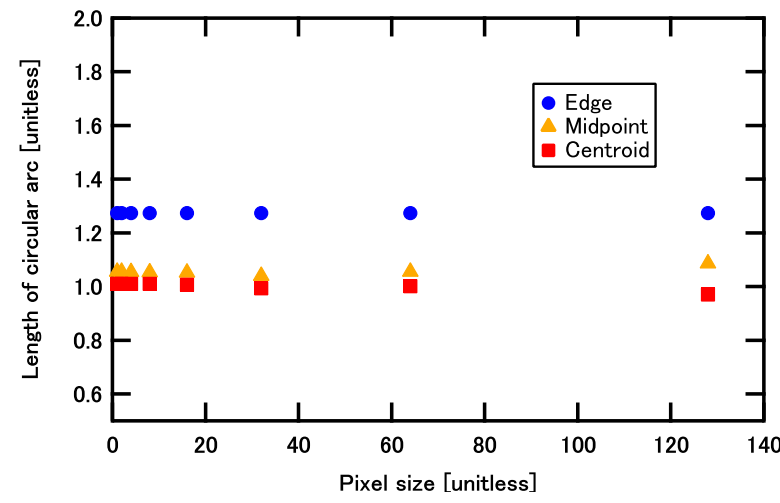

(a)

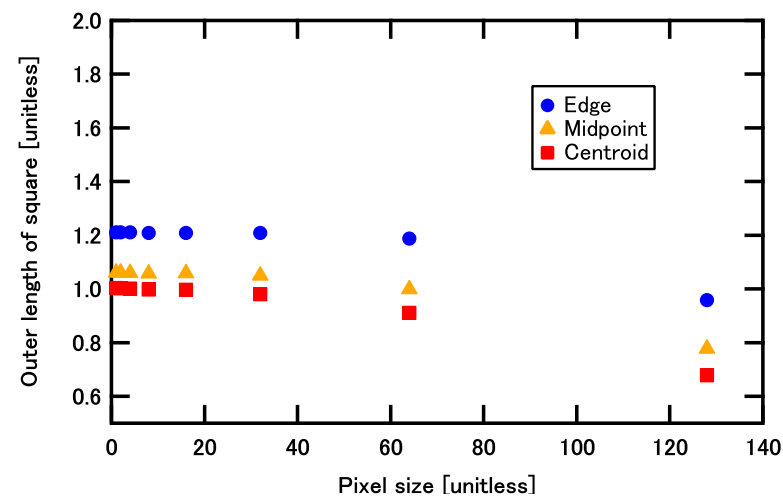

(c)

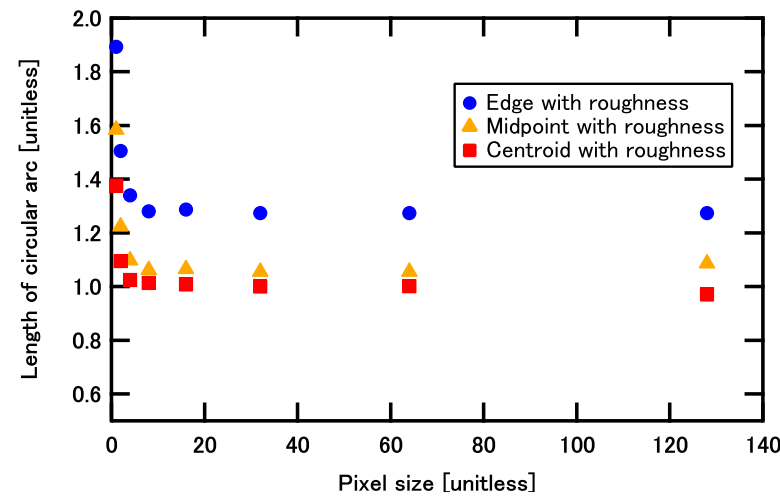

(b)

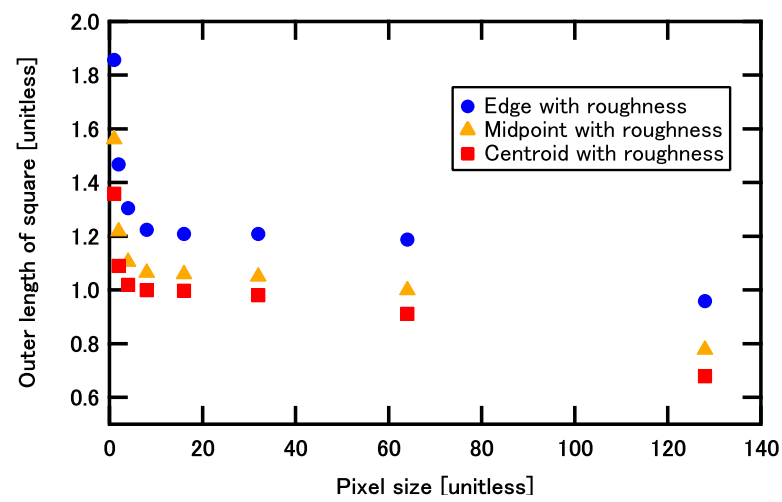

(d)

Figure 8: Calculated perimeter lengths against various resolutions. (a) Smooth circle, (b) circle with roughness, (c) smooth square and $(\mathrm{d})$ square with roughness

Based on the discussion described above, it is expected that precise values of TPB density and specific surface areas can be estimated in the case of FIB-SEM results as well by extrapolating the values from coarser resolutions. For the TPB density, values calculated by centroid method were used. TPB density and specific surface areas are extrapolated to zero pixel size by linear fitting from the values at pixel sizes of $20 \mathrm{~nm}$ to $40 \mathrm{~nm}$. The results are shown in Table 1 . In conventional SOFC microstructure reconstruction, typical pixel sizes of reconstruction are around 30 to $50 \mathrm{~nm}$. Therefore, TPB and surface area values at from the pixel size of $31.1 \mathrm{~nm}$ are shown for comparison. The values calculated from pixel size of $31.1 \mathrm{~nm}$ 
showed much smaller values than the extrapolated one, which indicates that higher resolution than voxel size of $31.1 \mathrm{~nm}$ is required for accurate estimation of TPB length and surface areas. The differences between the extrapolated values and values at voxel size of $31.1 \mathrm{~nm}$ were nearly $10 \%$ for TPB density and $5 \%$ for specific surface areas. Furthermore, the difference of TPB densities and specific surface areas between the extrapolated values and those calculated at vozel size of $98.5 \mathrm{~nm}$ were about $25 \%$ and $11 \%$, respectively. These results indicate that the dependence of microstructure on resolution cannot be ignored when we compare the results of different works with various resolutions. These differences would affect the result of electrochemical simulations in which the reaction rate is determined at TPB or surface. Therefore, it is recommended to use the data at several resolution to estimate the microstructure parameters accurately.

Table 1: Comparison of microstructure parameters with different estimation methods

\begin{tabular}{llll}
\hline Parameters & $\begin{array}{l}\text { Extrapolated value by } \\
\text { fitting from 20 nm to } \\
40 \mathrm{~nm} \text { pixel sizes }\end{array}$ & $\begin{array}{l}\text { Calculated value at } \\
\text { pixel size of } 31.1 \mathrm{~nm} \\
\text { (Relative value) }\end{array}$ & $\begin{array}{l}\text { Calculated value } \\
\text { pixel size of } 98.5 \mathrm{~nm} \\
\text { (Relative value) }\end{array}$ \\
\hline $\mathrm{TPB}\left[\mu \mathrm{m} \mu \mathrm{m}^{-3}\right]$ & $2.270(100 \%)$ & $2.066(91.01 \%)$ & $1.725(75.99 \%)$ \\
Ni Specific surface area & $1.114(100 \%)$ & $1.059(95.06 \%)$ & $0.9821(88.16 \%)$ \\
{$\left[\mu \mathrm{m}^{2} \mu \mathrm{m}^{-3}\right]$} & $2.487(100 \%)$ & $2.383(95.82 \%)$ & $2.208(88.78 \%)$ \\
$\begin{array}{l}\mathrm{YSZ} \mathrm{Specific} \mathrm{surface} \\
\text { area }\left[\mu \mathrm{m}^{2} \mu \mathrm{m}^{-3}\right]\end{array}$ & & & \\
\hline
\end{tabular}

\section{Conclusion}

Dependence of microstructure parameters on resolution was investigated using Ni-YSZ electrode microstructure which was reconstructed by high resolution FIB-SEM images with voxel sizes of $3 \mathrm{~nm}$. Calculated volume fractions showed almost constant value regardless of resolution. On the other hand, the values of TPB density and specific surface areas showed large values at high resolution. At coarser resolutions, those parameters showed moderate dependence on resolution. From the results of 3D median filtering and the analytical validations of $2 \mathrm{D}$ arc and square, large TPB and specific surface area values at high resolution are attributed to the roughness of the reconstructed structure in the stacking direction. This roughness originates from the stretch or shrinkage of the images due to the drift of the SEM and the fluctuations of the SEM position during FIB slicing. Accurate values of TPB density and specific surface areas were estimated by extrapolation from several values at coarser resolutions. The difference between estimated values and values at voxel sizes of $31.1 \mathrm{~nm}$, which is close to the typical voxel size of FIB-SEM reconstruction were nearly $10 \%$ for TPB and $5 \%$ for surface areas, respectively. Therefore, it is recommended to estimate the 
accurate values using the values at several resolutions. 


\section{Acknowledgements}

This work was partly supported by New Energy and Industrial Technology Development Organization (NEDO) and Japan Science and Technology Agency (JST). A part of this study was supported by NIMS Nanofabrication Platform in Nanotechnology Platform Project sponsored by the Ministry of Education,

Culture, Sports, Science and Technology (MEXT), Japan.

\section{References}

[1] Y.-c. Karen C.-Wiegart, J. S. Cronin, Q. Yuan, K. J. Y.-Kremski, S. A. Barnett, J. Wang, , J. Power Sources 218 (2012) $348-351$

[2] Y. Guan, Y. Gongc, W. Li, J. Gelb, L. Zhang, G. Liu, X. Zhang, X. Song, C. Xia, Y. Xiong, H. Wang, Z. Wu, Y. Tian, ,J. Power Sources 196 (2011) 10601-10605

[3] P.R. Shearing a, R.S. Bradley, J. Gelb, F. Tariq, P.J. Withers, N.P. Brandon, Solid State Ionics 216 (2012) 69-72

[4] J. Villanova, J. Laurencin, P. Cloetens, P. Bleuet, G. Delette H. Suhonen, F. Usseglio-Viretta, J Power Sources 243 (2013) 841-849

[5] Y. Guan, X. Dong, L. Zhang, G. Liu, Z. Liang, S. Chen, L. Chen, X. Zhang, Y. Xiong, H. Wang, Y. Tian, Int. J. Hydrogen Energy 41 (2016) 10833-10843

[6] J. R. Wilson, W. Kobsiriphat, R. Mendoza, H.-Y. Chen, J. M. Hiller, D. J. Miller, K. Thornton, P. W. Voorhees, S. B. Adler, S. A. Barnett, Nature Materials 5 (2006) 541-544

[7] H. Iwai, N. Shikazono, T. Matsui, H. Teshima, M. Kishimotoa, R. Kishida, D. Hayashi, K. Matsuzaki, D. Kanno, M. Saito, H. Muroyama, K. Eguchi, N. Kasagi, H. Yoshida, , J. Power Sources 195 (2010) 955-961

[8] T. Matsui, J.-Y. Kim, H. Muroyama, M. Shimazu, T. Abe, M. Miyao, K. Eguchi, Solid State Ionics 225 (2012) 50-54

[9] Z. Jiao, G. Lee, N. Shikazono, N. Kasagi, J. Electrochem. Soc. 159(7) (2012) F278-F286

[10] G. Brus, K. Miyoshi, H. Iwai, M. Saito, H. Yoshida, Int. J. Hydrogen Energy 40 (2015) 6927-6934

[11] F.Tariq, M.Kishimoto, V.Yufita, G.Cuia, M.Somalu, N.P.Brandon, J. Euro. Ceram. Soc. 34 (2014) 3755-3761

[12] N. Vivet, S. Chupina, E. Estradea, A. Richardb, S. Bonnamyc, D. Rochaisa, E. Brunetona, J. Power Sources, 196 (2011) 9989-9997

[13] L. Holzer, B. Munch, B. Iwanschitz, M. Cantoni, Th. Hocker, Th. Graule, J. Power Sources, 196 (2011) $7076-7089$

[14] T. Hutzenlaub, J. Becker, R. Zengerle, S. Thiele, ECS Electrochem. Letters 2(2) (2013) F14-F17

[15] M. Klingele, R. Zengerle, S. Thiele, J. Power Sources 275 (2015) 852-859

[16] J. Joos, T. Carraro, A. Weber, E. Ivers-Tiffee, J. Power Sources 196 (2011) 7302-7307

[17] Z. Chen, X. Wang, F. Giuliani, A. Atkinson, J. Power Sources 273 (2015) 486-494

[18] W. M. Harris, J. J. Lombardo, G. J. Nelson, B. Lai, S. Wang, J. Vila-Comamala, M. Liu, M. Liu, W. K. S. Chiu, Sci. Rep 4 (2014) 5246

[19] T. Shimura, Z. Jiao, S. Hara, N. Shikazono, J. Power Sources 267 (2014) 58-68

[20] S. Hara, A. Ohi, N. Shikazono, J. Power Sources 276 (2015) 105-112

[21] P. Thevenaz, U.E. Ruttimann, M. Unser, IEEE Trans. Image Process. 7(1) (1998) 27-41

[22] N. Shikazono, D. Kanno, K. Matsuzaki, H. Teshima, S. Sumino, N. Kasagi, J. Electrochem. Soc. 157(5) (2010) B665-B672 
[23] K. Matsuzaki, N. Shikazono, N. Kasagi, J. Power Sources 196 (2011) 3073-3082

[24] A.Z. Lichtner, D. Jauffres, D. Roussel, F. Charlot, C.L. Martin, R.K. Bordia, J. Euro. Ceram. Soc. 35 (2015) 585-595

[25] N. S. K. Gunda, H.-W. Choi, A. Berson, B. Kenney, K. Karan, J. G. Pharoah, S. K. Mitra, J. Power Sources 196 (2011) $3592-3603$ 